ORIGINAL

\title{
Estilos de vida, sobrepeso y obesidad en adolescentes de enseñanza media de La Habana
}

\section{Consuelo Macías Matos*, María Elena Díaz Sánchez, Gisela María Pita Rodríguez, Beatriz Basabe Tuero, Dania Herrera Javier y Vilma Moreno López}

Instituto de Nutrición e Higiene de los Alimentos (INHA)

Recibido el 4 de noviembre de 2011; aceptado el 21 de diciembre de 2011

\author{
PALABRAS CLAVE \\ Peso corporal; \\ Sobrepeso; \\ Composición corporal; \\ Adolescentes; \\ Actividad física; \\ Estilo de vida \\ sedentario; \\ Hábitos alimentarios; \\ Hábitos de ingesta
}

\begin{abstract}
Resumen
Introducción: La elevada prevalencia de sobrepeso y obesidad constituye un problema de salud en Cuba y no hay suficiente información sobre adolescentes. El objetivo del presente estudio es evaluar la relación entre el exceso de peso y adiposidad con la actividad física, las horas de televisión y los hábitos alimentarios en adolescentes.

Material y métodos: Se estudió a 604 adolescentes (edad, 12 años) de La Habana. Se analizaron: índice de masa corporal, composición corporal, antecedentes de obesidad en la niñez, actividad física, horas viendo televisión, consumo, hábitos y preferencias alimentarias. Se realizó estadística descriptiva y de asociación. Para el análisis de regresión logística se utilizó el exceso de peso y de adiposidad como variables dependientes.

Resultados: El exceso de peso corporal fue más frecuente en los varones. Se encontró una alta prevalencia de exceso de adiposidad, el $49,8 \%$ en mujeres y el $37,8 \%$ en varones. Los varones $(61,7 \%)$ fueron más activos que las mujeres $(33,6 \%)$. De lunes a viernes, el $46,7 \%$ de los varones y el $55,9 \%$ de las mujeres dedicaron más de 2 h/día a ver televisión, que aumentó a más del $75 \%$ en ambos sexos los sábados y domingos. Realizar menos de 5-6 comidas/día duplicó el riesgo de tener exceso de peso; el sobrepeso corporal en la niñez también fue un factor de riesgo. No desayunar incrementó el riesgo de adiposidad casi al doble.

Conclusiones: El exceso de peso, y especialmente el de adiposidad, puede ser considerado un problema de salud en los adolescentes estudiados. Se observó un consumo demasiado alto de azúcar, un tiempo excesivo viendo la televisión e insuficiente actividad física.

(c) 2011 Asociación Española de Dietistas-Nutricionistas. Publicado por Elsevier España, S.L. Todos los derechos reservados.
\end{abstract}

*Autor para correspondencia

Correo electrónico: cmacias@infomed.sld.cu (C. Macías Matos). 
KEYWORDS

Body weight;

Overweight;

Body composition;

Adolescents;

Physical activity;

Sedentary life style;

Dietary habits;

Eating habits

\section{Lifestyle, Overweight and Obesity in a Population of Adolescents From Havana}

\begin{abstract}
Introduction: The high prevalence of overweight and obesity is a health problem in Cuba, and there is a lack of information on adolescents. The objective of the present study was to evaluate the relationship between excessive weight and adiposity with physical activity, hours of television (TV), and eating habits in adolescents.

Material and methods: A total of 604 adolescents (12 years) from Havana were studied. The variables analysed included, body mass index, body composition, history of obesity in infancy, physical activity, hours watching television, eating habits and dietary preferences. A descriptive and correlation analysis was performed. In the logistic regression analysis, excess weight and adiposity were used as dependent variables.

Results: Excess body weight was more common in boys. There was high prevalence of excess adiposity, $49.8 \%$ in girls and $37.8 \%$ in boys. The boys $(61.7 \%)$ were more active than the girls (33.6\%). Just under half $(46.7 \%)$ of the boys, and $55.9 \%$ of the girls spent more than 2 hours/day watching television, which increased to more than $75 \%$ for both sexes on Saturdays and Sunday. Making less than 5-6 meals/day doubled the risk of being overweight. Body overweight in infancy was also a risk factor. Not having breakfast increased the risk of adiposity by almost double.

Conclusions: Excess weight, and particularly excess adiposity, may be considered a health problem in the adolescents studied. A very high consumption of sugar, an excessive time watching TV, and insufficient physical activity was observed.

(c) 2011 Asociación Española de Dietistas-Nutricionistas. Published by Elsevier España, S.L. All rights reserved.
\end{abstract}

\section{Introducción}

El exceso de peso corporal y de adiposidad constituye una de las principales amenazas para la salud en la mayoría de los países y es un factor de riesgo predisponente de diabetes mellitus tipo 2, enfermedades cardiovasculares, hipertensión y cáncer ${ }^{1}$. Su tendencia creciente en las últimas décadas en niños y adolescentes cubanos ${ }^{2,3}$ influirá en el incremento de las enfermedades crónicas no transmisibles en el país.

Entre los factores asociados con la obesidad, se encuentran algunos no modificables como los genéticos, mientras que sobre otros se pueden realizar acciones encaminadas a alcanzar un mejor estado de salud; estos son los relacionados con el estilo de vida. Entre ellos, los principales contribuyentes de la obesidad en la niñez y adolescencia son la insuficiente actividad física, el excesivo tiempo dedicado a ver televisión (TV), vídeos o videojuegos y otros entretenimientos pasivos y el elevado consumo de bebidas azucaradas y de alimentos de alta densidad energética ${ }^{4-7}$.

La adolescencia es una etapa vulnerable de la vida, fuertemente influida por características del macroentorno y el microentorno ${ }^{8}$; la ingesta energética elevada y las necesidades de nutrientes incrementadas en este grupo pueden contribuir significativamente al desarrollo de sobrepeso corporal cuando se eleva el consumo de alimentos ricos en calorías y el gasto energético es deficiente. La inactividad física y los hábitos dietéticos inadecuados han sido identificados como factores predisponentes de la obesidad con gran impacto en el desarrollo de enfermedades crónicas en la adultez $z^{1,8}$. Las acciones que se tomen para modificar estilos de vida en la adolescencia contribuirán a detener la epidemia de obesidad a corto y largo plazo.
El propósito del presente trabajo es evaluar la relación del sobrepeso corporal con la actividad física, las horas dedicadas a ver TV y el consumo, los hábitos y las preferencias alimentarias en adolescentes habaneros. Este estudio se realizó como parte de un diagnóstico inicial orientativo para la aplicación de un modelo de intervención nutricional.

\section{Materiales y métodos}

\section{Participantes}

Se realizó un estudio descriptivo transversal en el universo de adolescentes de $7 .^{\circ}$ grado de cuatro escuelas secundarias básicas (enseñanza media básica) del municipio Habana Vieja, en la ciudad de La Habana. Participaron voluntariamente (sin asignación aleatoria) 604 adolescentes (313 mujeres y 291 varones) de 12 (media, 12,1 \pm 0,37; mediana, 12) años de edad.

\section{Antropometría y composición corporal}

Se realizó una evaluación antropométrica que incluyó las mediciones de peso, estatura, circunferencia del brazo y pliegues tricipital y subescapular, según las recomendaciones del Programa Biológico Internacional ${ }^{9}$. Con ellos se calcularon el índice de masa corporal (IMC), la masa muscular y el porcentaje de grasa corporal total (adiposidad total), este último con las ecuaciones de Boileau et $\mathrm{al}^{10}$. Se utilizaron los puntos de corte del IMC para la edad (< p10, bajo peso; p10-90, peso normal; > p90, exceso de peso), área muscular del brazo (< p10, masa muscular deficiente; p10- 
90, masa muscular normal; > p90, masa muscular elevada), correspondientes a las curvas nacionales de la población cubana $^{11,12}$. Los niveles de clasificación del porcentaje de grasa corporal total se obtuvieron según los criterios de Deurenberg et $\mathrm{al}^{13} \mathrm{y}$ se agruparon en las categorías de grasa deficiente, adecuada y excesiva. Para el análisis se utilizó la categoría de sobrepeso global derivada del IMC que abarca el sobrepeso y la obesidad.

Se registró el antecedente patológico personal de sobrepeso corporal global en la niñez.

\section{Actividad física}

La actividad física se estimó por encuesta dirigida personalmente al adolescente, con criterio de saturación de la información, teniendo en cuenta las actividades escolares (educación física, deporte) las actividades extraescolares vigorosas (fútbol, karate, taekwondo) y moderadas (juegos de pelota, bicicleta, patines, gimnasia, bailes) y, por último, caminatas en los 7 días de la semana. Los datos se computaron tomando como referencia la encuesta corta del $\mathrm{IPAQ}^{14}$ y se clasificaron en sedentarios, insuficientemente activos, activos y muy activos. Se registró el número de horas dedicadas a ver TV, vídeos, usar la computadora y videojuegos y otros juegos de mesa, que conformaron la variable "horas viendo TV", que se analizó por separado de lunes a viernes y los fines de semana. Según el tiempo dedicado a estas actividades, se realizó la siguiente clasificación: poco (0-1 h), adecuado (2 h) y mucho (> $2 \mathrm{~h})$.

\section{Hábitos alimentarios}

De las encuestas de frecuencia de consumo de alimentos, hábitos, gustos y preferencias alimentarias descritas en detalle en una publicación anterior ${ }^{15}$, se analizaron para este trabajo las de interés en relación con el sobrepeso y la obesidad, como el consumo de azúcar y frutas y hortalizas, el hábito de desayunar y el número de comidas diarias.

\section{Análisis de los datos}

La estadística descriptiva para cada variable se realizó con el paquete estadístico SPSS versión 16.0. Se obtuvieron las tablas de contingencia con la prueba de la $\chi^{2}$ para analizar asociaciones entre las variables, estratificando por sexo y con umbral de significación estadística en $p<0,05$.

Para evaluar integralmente los factores estudiados antes, se realizó el análisis de regresión logística (backward method). Se tomaron como variables dependientes el IMC (sobrepeso+obeso/bajopeso+desnutrido+normopeso) y la adiposidad (grasa excesiva/grasa normal+grasa deficiente), y como variables independientes se incluyeron sexo, antecedentes de exceso de peso en la niñez, nivel de actividad física, nivel de sedentarismo expresado como horas viendo TV, hábito de desayunar, número de comidas al día y frecuencia de consumo de frutas y verduras. De acuerdo con los resultados encontrados, con las horas de TV el fin de semana se tomó el valor de la mediana para dicotomizar la variable. Los resultados se presentan como razón de disparidad (OR) e intervalo de confianza del 95\% (IC95\%).

\section{Resultados}

\section{Estado nutricional}

En la tabla 1 se presentan las características antropométricas de los adolescentes por sexo. El dimorfismo sexual es evidente en las mediciones e índices antropométricos y de la composición corporal. Los varones tienen menores peso y estatura que las mujeres, pero mayor área muscular del brazo; las mujeres tienen mayores circunferencia del brazo y adiposidad, reflejada en los pliegues cutáneos y la estimación de la grasa corporal total.

En el análisis de la distribución de frecuencia de los indicadores antropométricos, se encontró exceso de peso corporal en el $29,2 \%$ de los varones y el $20,3 \%$ de las mujeres, de acuerdo con los puntos de corte del IMC. La masa muscular es mayor en los varones $(21,2 \%)$ que en las mujeres $(8,3 \%)$. El porcentaje de grasa muestra alta prevalencia de valores excesivos tanto en varones $(37,8 \%)$ como en mujeres $(49,8 \%)$.

La figura 1 integra los tres indicadores, y se puede observar que sólo una tercera parte del grupo se clasificó como normal por antropometría y composición corporal (el 36,5\% de los varones y el $35,3 \%$ de las mujeres). En el cuadrante correspondiente a masa muscular e IMC normal, el 14,9\% de los varones y el $29,6 \%$ de las mujeres tenían exceso de grasa.

Haber sido un niño con sobrepeso u obeso se asoció significativamente con el sobrepeso corporal para el total de los adolescentes $\left(\chi^{2}=9,59 ; p=0,008\right)$ y para los varones $\left(\chi^{2}=9,37 ; p=0,009\right)$, pero no con la adiposidad $\left(\chi^{2}=4,18\right.$; $\mathrm{p}=0,124)$.

\section{Actividad física}

La figura 2 muestra la distribución porcentual de la actividad física por categorías estratificada por sexo. Los varones resultaron ser más activos que las mujeres $\left(\chi^{2}=72,36\right.$; $\mathrm{p}<0,001)$. El $61,7 \%$ de los primeros clasificaron como activos y muy activos; por el contrario, el $66,4 \%$ de las mujeres clasificaron como sedentarias e insuficientemente activas. No se

Tabla 1 Estadística descriptiva de las variables antropométricas entre los adolescentes de La Habana Vieja

\begin{tabular}{lcc}
\hline & Mujeres & \multicolumn{1}{c}{ Varones } \\
\hline Edad (años) & $12,1 \pm 0,35$ & $12,1 \pm 0,39$ \\
Peso $(\mathrm{kg})$ & $45,17 \pm 10,30$ & $43,89 \pm 10,23$ \\
Estatura $(\mathrm{cm})$ & $153,33 \pm 6,82$ & $152,66 \pm 8,92$ \\
Pliegue del tríceps $(\mathrm{mm})$ & $16,22 \pm 7,16$ & $12,90 \pm 6,88$ \\
Pliegue subescapular $(\mathrm{mm})$ & $13,81 \pm 7,28$ & $10,96 \pm 6,96$ \\
Circunferencia del brazo & $22,21 \pm 3,33$ & $22,05 \pm 3,18$ \\
(cm) & & \\
Área muscular del brazo & $23,59 \pm 5,18$ & $26,11 \pm 6,23$ \\
(cm $)$ & & \\
Índice de masa corporal & $19,12 \pm 3,72$ & $18,67 \pm 3,19$ \\
Masa grasa (\%) & $25,05 \pm 6,51$ & $18,86 \pm 7,51$ \\
\hline
\end{tabular}

Las cifras expresan media \pm desviación estándar. 
Mujeres

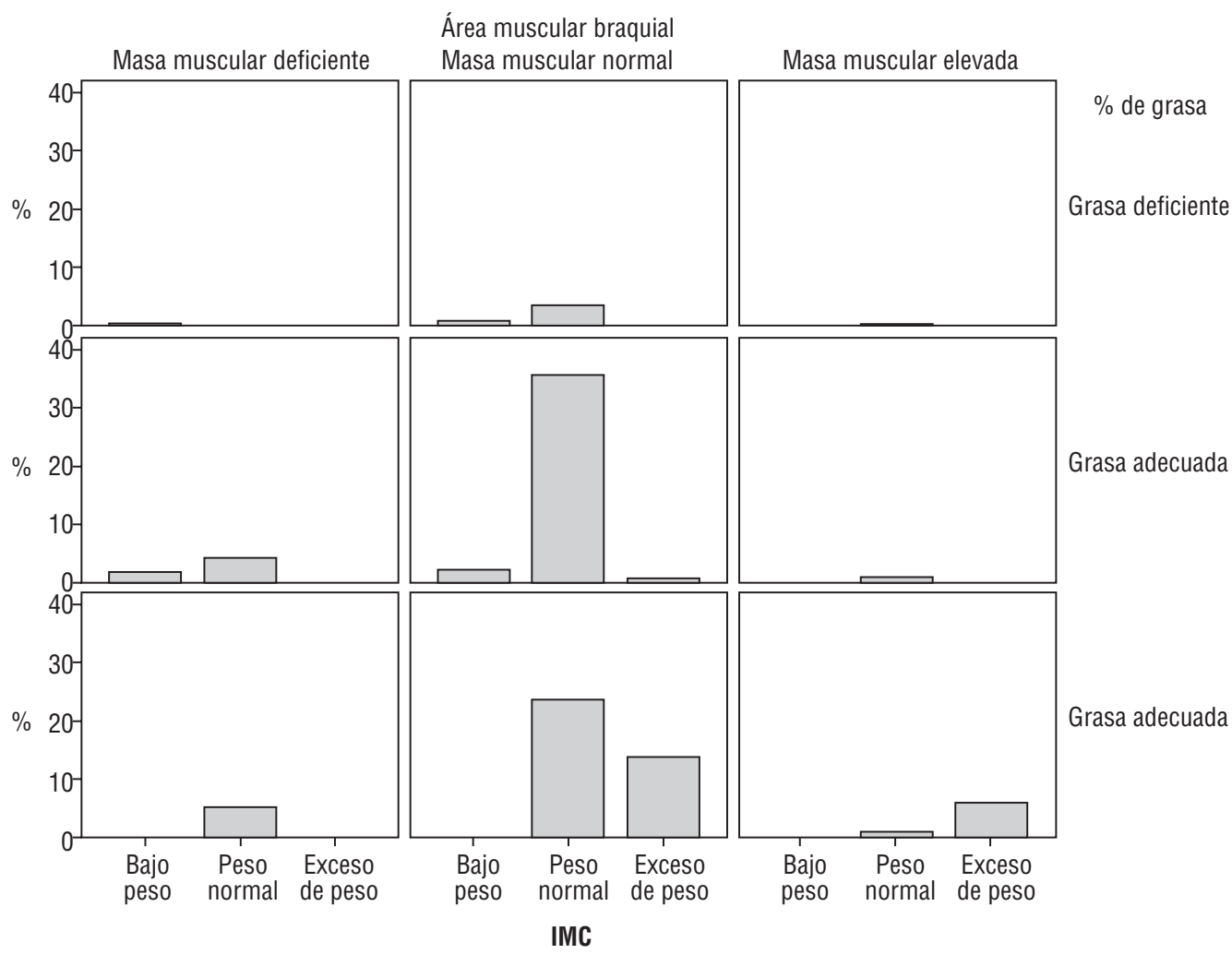

Varones

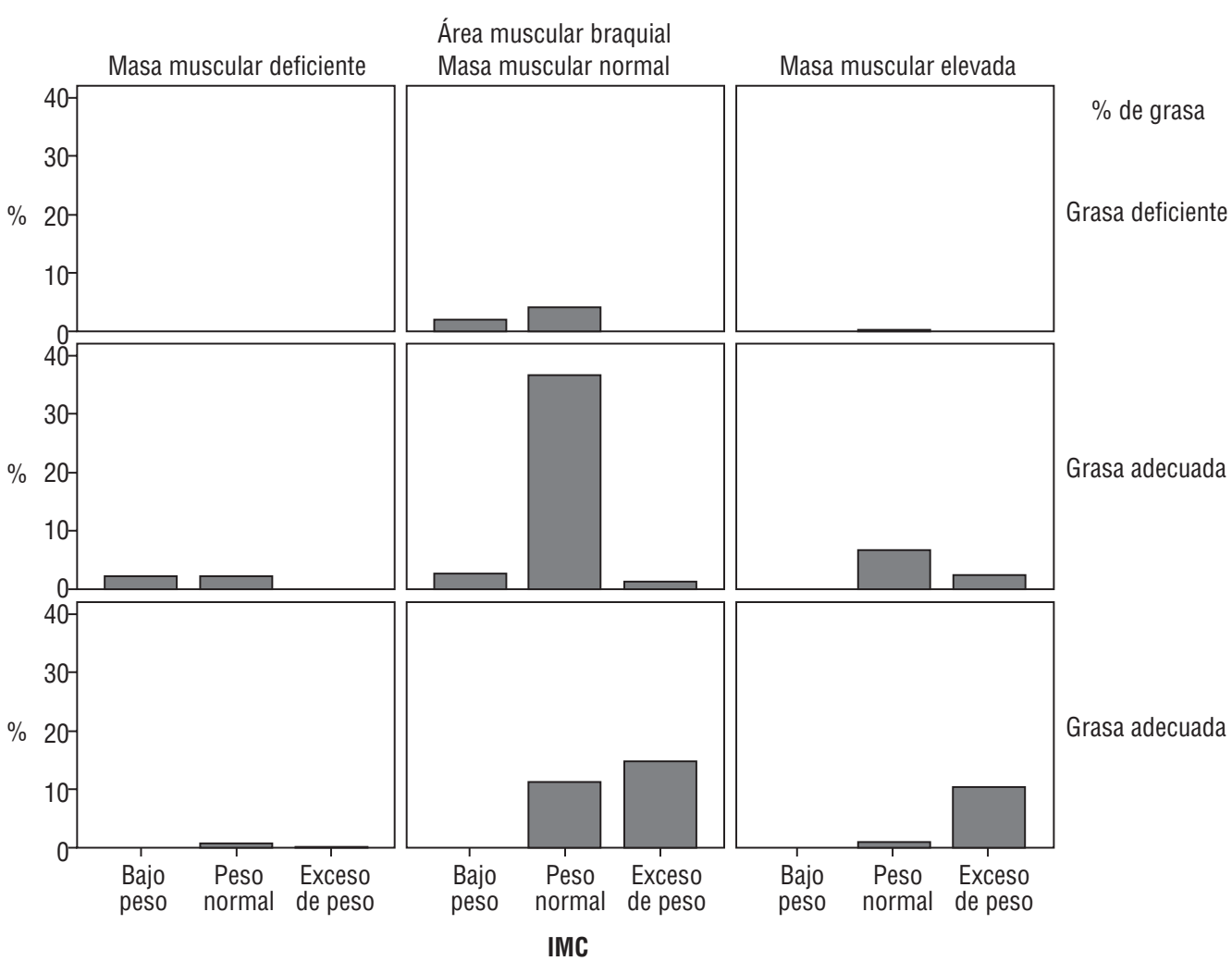

Figura 1 Distribución porcentual entre el índice de masa corporal (IMC), la adiposidad total y la masa muscular, por sexo, entre adolescentes de La Habana Vieja. 


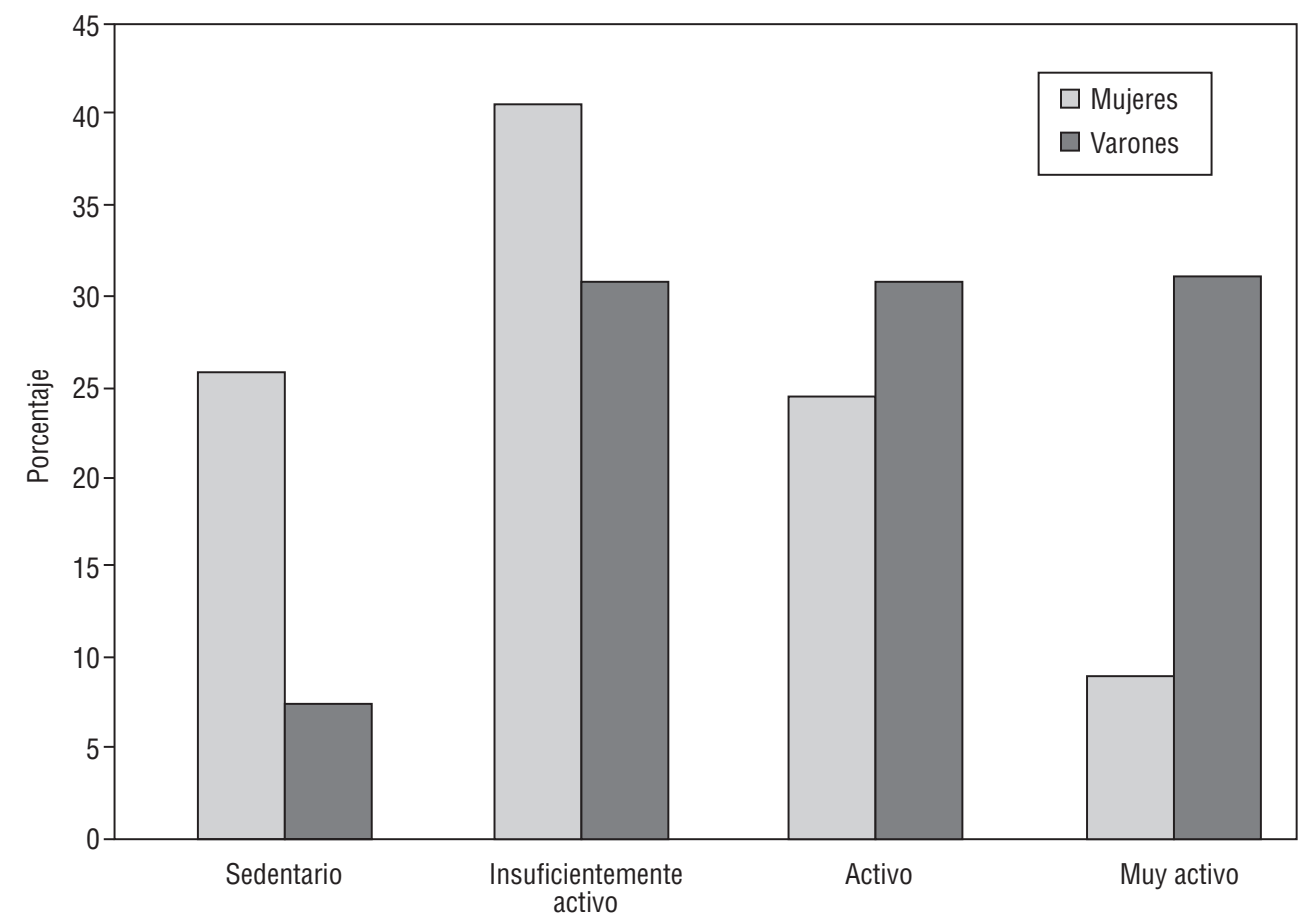

Figura 2 Distribución porcentual de la actividad física (AF) por sexo entre adolescentes de La Habana Vieja.

Tabla 2 Distribución porcentual y valores medios de las horas dedicadas a ver televisión, por sexo, entre los adolescentes de La Habana Vieja

\begin{tabular}{lllll}
\hline & Poco $(0-1 \mathrm{~h}), \%$ & Adecuado $(2 \mathrm{~h}), \%$ & Mucho $(>2 \mathrm{~h}), \%$ & Ver TV $(\mathrm{h})$, mediana \\
\hline Lunes a viernes & & & & 2 \\
$\quad$ Varones & 16,3 & 37,1 & 46,6 & 3 \\
$\quad$ Mujeres & 8,4 & 34,6 & 57,0 & 4 \\
Sábados y domingos & & & 79,2 & 4 \\
$\quad$ Varones & 6,0 & 14,8 & 77,7 & \\
$\quad$ Mujeres & 8,2 & 14,1 &
\end{tabular}

encontró asociación entre las categorías de la actividad física y el IMC, pero sí con el nivel de adiposidad para el total de adolescentes $\left(\chi^{2}=23,06 ; p=0,001\right)$ y para los varones $\left(\chi^{2}=20,68 ; p=0,002\right)$. En las mujeres se observó tendencia a que las que tenían exceso de grasa fueran menos activas.

Respecto al tiempo dedicado a actividades sedentarias (tabla 2), representadas principalmente por horas dedicadas a ver TV, se encontró un comportamiento diferente para varones y mujeres entre semana y semejante el fin de semana. De lunes a viernes, las mujeres veían más TV que los varones $\left(\chi^{2}=13,19 ; p=0,001\right)$. No se encontraron diferencias por sexo en los fines de semana $\left(\chi^{2}=1,08 ; p=0,582\right)$. De lunes a viernes, aproximadamente la mitad de los adolescentes (el $46,7 \%$ de los varones y el $55,9 \%$ de las mujeres) dedicaban más de $2 \mathrm{~h} /$ día a actividades sedentarias en horario extraescolar y adicionales a las horas que estaban atendiendo a clases. Los sábados y domingos, este tiempo llega a más de tres cuartos del grupo de estudio para los dos sexos.
Al analizar las horas dedicadas a actividades sedentarias (entre semana y fin de semana) con las variables antropométricas, no se encontró asociación estadísticamente significativa con el IMC (lunes a viernes, $\chi^{2}=2,06 ; p=0,724$; sábado y domingo, $\left.\chi^{2}=1,45 ; p=0,836\right)$ ni con el nivel de adiposidad total (lunes a viernes, $\chi^{2}=2,12 ; p=0,714$; sábado y domingo, $\chi^{2}=5,23 ; p=0,265$ ).

Cuando se realizó el análisis por sexo de las horas dedicadas a ver TV entre semana y los fines de semana con las categorías de la actividad física, no se encontró asociación estadísticamente significativa.

\section{Hábitos y consumo de alimentos}

El $84,9 \%$ de los adolescentes desayunaba. El 48,4\% realizaba entre 5 y 6 comidas al día y el $48,1 \%$, entre 3 y 4 . No se encontró asociación entre el hábito de desayunar y el IMC $\left(\chi^{2}=3,30 ; p=0,192\right)$, pero sí con la adiposidad para el total 
Tabla 3 Resultados del último paso de la regresión logística (backward method) para la asociación del IMC y la adiposidad (variables dependientes) con el sexo, la actividad física, las horas viendo TV, el desayuno, el número de comidas por día, consumo de frutas, consumo de verduras y obesidad en la niñez

\begin{tabular}{llll}
\hline IMC a & & Adiposidad $^{\mathrm{b}}$ & \\
\hline Variables independientes & OR (IC95\%) & Variables independientes & OR (IC95\%) \\
Sexo & $0,52(0,34-0,79)$ & Actividad física & $1,41(0,98-2,03)$ \\
Obesidad en la niñez & $1,69(1,11-2,59)$ & Horas TV sábado-domingo & $1,41(0,97-2,04)$ \\
Número de comidas diarias & $2,08(1,35-3,22)$ & Desayunar & $1,91(1,14-3,22)$ \\
\hline
\end{tabular}

aVariable dependiente: sobrepeso + obeso / bajo peso + desnutrido + normopeso. Total de casos, $\mathrm{n}=577$.

bVariable dependiente: exceso de grasa / grasa adecuada + deficiente. Total de casos, $n=579$.

Variables de entrada en el Paso 1: Sexo (mujeres/varones), obesidad en la niñez (sí/no), actividad física (sedentario + insuficientemente activo/activo + muy activo), horas de TV entre semana $(>2 \mathrm{~h} / \leq 2 \mathrm{~h}$ ) y sábado-domingo ( $\geq 4 \mathrm{~h} /<4 \mathrm{~h})$, hábito de desayuno (no/sí), número de comidas al día (1-4/5-6) y consumo de frutas y verduras (< 3 alimentos 3 días por semana/> 3 alimentos 3 días por semana).

$\left(\chi^{2}=11,45 ; p=0,003\right)$ y para las mujeres $\left(\chi^{2}=12,13\right.$ $p=0,002)$. Se encontró asociación entre el número de comidas al día y el IMC para el total $\left(\chi^{2}=18,61 ; p=0,017\right)$ y para las mujeres $\left(\chi^{2}=23,82 ; p=0,002\right)$, pero no con la adiposidad $\left(\chi^{2}=5,12 ; p=0,745\right)$.

El consumo promedio de pan fue de $200 \mathrm{~g} /$ día (80-800 $\mathrm{g} /$ día). La mayoría de los adolescentes (> 95\%) refirieron comer arroz diariamente y frecuentemente ( $>3$ veces/semana) refrescos, pizzas, alimentos fritos, dulces y alimentos "callejeros". La ingesta de estos alimentos se estimó mediante encuesta de frecuencia de consumo, que no tiene la exactitud requerida para someterla a un análisis estadístico en búsqueda de relación con el IMC o la adiposidad total.

De las bebidas azucaradas carbonatadas, sólo se tuvo en consideración su frecuencia de consumo, que fue muy elevada. Por la costumbre cubana de consumir elevadas cantidades de azúcar añadido a líquidos, estas se cuantificaron. De los adolescentes que participaron en el estudio, solamente un $61-77 \%$ dio respuesta a la cantidad de azúcar que añadía a los líquidos, ya que en muchos casos los alimentos eran preparados por los familiares y ellos desconocían la forma de preparación. Entre un 70 y un $78 \%$ de los adolescentes que dieron respuesta a esta pregunta añadían dos o más cucharadas de azúcar (sacarosa principalmente refinada) a un vaso de leche, jugos de frutas, refrescos instantáneos y yogur de soja (los dos últimos contienen azúcar en su procesamiento industrial). Solamente menos de un $5 \%$ ingería estas bebidas sin añadir azúcar. No se encontró asociación entre la cantidad de azúcar añadida y el IMC o la adiposidad.

\section{Resultados de la regresión logística}

En la tabla 3 se resumen los resultados encontrados en el análisis del último paso de la regresión logística.

Las variables asociadas significativamente con el sobrepeso y la obesidad son el sexo masculino, los antecedentes de obesidad en la niñez y hacer menos de 5 comidas al día. No alcanzar las recomendaciones de 5-6 comidas al día duplicó el riesgo de tener exceso de peso.
En cuanto a la adiposidad, las variables asociadas difieren a las encontradas en el IMC; sólo el hábito de no desayunar tuvo significación estadística incrementando el riesgo casi al doble. Las variables actividad física y horas viendo TV los fines de semana estuvieron asociadas, pero sin significación estadística.

\section{Discusión}

La elevada prevalencia de exceso de peso y adiposidad encontrada en el presente estudio está acorde con los resultados del estudio de Esquivel y González del año 2005 en niños y adolescentes habaneros ${ }^{2}$, en quienes el exceso de grasa $(28,8 \%)$ fue mucho mayor que el exceso de peso $(16,5 \%)$, de forma tal que parte de los que clasificaron con IMC normal tenían exceso de grasa. Estos autores encontraron una tendencia en aumento (1972-2005) del exceso de peso, más pronunciada aún para la adiposidad. Esta tendencia la refieren Ogden et $\mathrm{al}^{16}$ en adolescentes de Estados Unidos y Moreno et $\mathrm{al}^{17}$ en adolescentes españoles; en estos fue mayor el incremento en el exceso de peso que en la grasa. Asimismo, en un estudio realizado recientemente en 30 países europeos se encontraron diferencias en la prevalencia de sobrepeso y obesidad entre regiones y una tendencia en aumento, aunque con una estabilización en los últimos años en algunos pocos países ${ }^{18}$.

No resulta fácil establecer comparaciones con estudios en otros países, ya que los diferentes autores emplean distintos valores de referencia y puntos de corte correspondientes tanto a patrones locales como internacionales, que tienen interpretaciones diferentes para realizar las clasificaciones del estado nutricional; los primeros describen el comportamiento de los individuos respecto a su población, mientras que los segundos proporcionan una meta a alcanzar. No obstante, en todas las publicaciones recientes de distintos países y áreas del mundo se comunica una elevada prevalencia de sobrepeso y obesidad, que para algunos alcanza entre una quinta y una cuarta parte de los adolescentes $^{5,17,19-22}$, y en los peores casos hasta un tercio ${ }^{16,23-25}$. La mayoría de estos estudios no tienen correspondencia entre 
sí en cuanto al muestreo ni a la representatividad de país, y esto puede crear un sesgo en el dato de las tan elevadas prevalencias, lo cual no restaría alarma a la epidemia de obesidad que enfrenta el mundo actualmente. En general, los informes indican que las prevalencias de sobrepeso y obesidad son más altas en los varones que en las mujeres. En los casos en que, además del exceso de peso, se analizó la adiposidad, las cifras se invierten y muestran en las mujeres más exceso de grasa que en los varones ${ }^{5,17,20,25}$, lo cual coincide con los resultados del presente estudio.

Las relaciones entre el peso y la talla no son suficientes para diagnosticar un exceso de peso explicado como grasa. Diferentes informes han demostrado que un elevado IMC puede explicarse en algunos individuos por un mayor peso de la región del tronco respecto a las extremidades inferiores, mientras que en otros contribuye más la longitud de las piernas sin que esto implique una mayor adiposidad, y a que el índice no considera el concurso de la adiposidad central ${ }^{17,26,27}$. De esta manera, el IMC no da una visión completa del estado nutricional de un adolescente, que puede ser tributario de la grasa, el músculo o la talla, por lo que la evaluación de la composición corporal es siempre necesaria para completar el diagnóstico individualizado.

En cuanto al dimorfismo sexual, los varones de este estudio tuvieron valores menores en la mayoría de las dimensiones corporales, a excepción de la masa muscular. Las mujeres usualmente tienen más adiposidad que los varones, pero las mayores cifras de peso y estatura que ellas presentaban son el resultado de una maduración biológica que antecede a la de los varones; cuando ellas están aproximadamente en el pico de crecimiento de la adolescencia (12 años como término medio), los varones aún no han comenzado y, por lo tanto, sus características corporales son menos desarrolladas.

En la literatura se plantea que el exceso de peso en la adultez tiene su antecedente en la niñez y la adolescencia ${ }^{1}$; esto concuerda con la relación encontrada entre el antecedente de exceso de peso en la niñez y el sobrepeso corporal en los adolescentes estudiados.

La actividad física es una vía efectiva para mantener la salud física y mental, además de contribuir a la prevención de las enfermedades crónicas y la discapacidad. Entre los beneficios que aporta, están la reducción y el mantenimiento del peso corporal, la mejora del perfil lipídico y la sensibilidad a la insulina y la mejora de la función cognitiva y la autoestima. El desarrollo tecnológico y los cambios demográficos han creado un "ambiente obesogénico" caracterizado por la disminución de la actividad física y el establecimiento de estilos de vida más sedentarios. En estos ambientes se encuentran "atrapados" los niños y jóvenes con la TV, el vídeo, el uso de la computadora y sus juegos virtuales. En algunas ciudades la inseguridad ciudadana disminuye la posibilidad de los juegos al aire libre y el uso de las bicicletas o caminatas para ir y regresar de la escuela ${ }^{28}$. Por otra parte, existe un programa de desarrollo de ciudades saludables, promocionado por la OPS ${ }^{29}$ con el objetivo de incrementar la posibilidad de que la población realice actividades físicas.

La Habana Vieja es la zona antigua de la capital y es compacta, de modo que la disponibilidad de espacios públicos cualificados para el desarrollo de actividades físicas al aire libre es limitado. No obstante, los niños y adolescentes acostumbran ir a la escuela caminando y jugar al aire libre con seguridad. Lo primero es válido para los dos sexos, pero lo segundo tiene diferencias de sexo que pueden deberse a las tradiciones culturales; mientras que los varones juegan en la calle practicando deportes y juegos que clasifican como actividad física moderada y vigorosa, las mujeres permanecen en casa y, la mayor parte del tiempo, dedicadas a actividades sedentarias. Esto se corresponde con el mayor número de horas que ven TV las mujeres entre semana, el predominio de varones activos y muy activos y el mayor exceso de grasa encontrado en las mujeres. Estos resultados coinciden con los niveles de actividad física registrados en niñas cubanas de edad preescolar ${ }^{30}$.

Algunos autores son del criterio que, de las intervenciones dedicadas a la prevención y tratamiento de la obesidad, las que se ocupaban de disminuir las horas de actividades sedentarias tuvieron un efecto más importante en la ganancia de peso y el exceso de grasa que las que trataban de aumentar la actividad física ${ }^{25,31}$. Según la revisión realizada por De Mattia ${ }^{4}$, todas las intervenciones dedicadas a disminuir comportamientos sedentarios en niños y adolescentes condujeron a mejorar los parámetros antropométricos.

Existen criterios contradictorios entre las horas dedicadas a ver la TV y la actividad física. Olivares et $\mathrm{al}^{23}$ consideran que ambas están relacionadas. Por el contrario, Taveras et $\mathrm{al}^{32}$ no encontraron asociación entre las horas viendo TV y la actividad física moderada y vigorosa, y recomendaron que en los programas de salud diseñados para el abordaje de la obesidad se las considere dos actividades independientes determinantes del peso corporal. En la experiencia de Sisson et a $^{33}$, la influencia combinada de bajos niveles de actividad física y gran cantidad de horas de TV se asoció significativamente con alto riesgo de sobrepeso en ambos sexos. Como el IMC no mide con exactitud las características del sobrepeso, sería más prudente utilizar indicadores que estimen la adiposidad en asociación con el grado de sedentarismo o de actividad física. En el presente estudio sí se encontraron asociaciones de la actividad física y el exceso de grasa.

De los resultados del estudio HELENA, llevado a cabo en 10 países europeos, se pudo concluir que $60 \mathrm{~min} /$ día de actividad física moderada y vigorosa se asocian con una reducción del riesgo de sobrepeso y obesidad en los adolescentes ${ }^{5}$ y que la actividad física vigorosa parece ser el factor clave para disminuir la posibilidad de ser obeso.

La TV, que es a lo que más tiempo dedican los adolescentes entre las actividades sedentarias, tiene doble efecto negativo: la inactividad física en sí y la publicidad de alimentos que generalmente no son de valor nutricional. Estos anuncios tienen un efecto importante en las preferencias y la selección de los alimentos, especialmente en niños y jóvenes $^{31,32,34}$. Los expertos recomiendan para los niños y adolescentes no más de $2 \mathrm{~h}$ diarias ${ }^{7}$. La TV cubana no induce al consumo de alimentos inapropiados, ya que está libre de esta propaganda y, por el contrario, es parte importante de un Programa Nacional de Cultura Alimentaria que promueve una alimentación sana y adecuada.

Otro determinante esencial entre los estilos de vida es los hábitos dietéticos, y entre ellos los que más repercuten en los adolescentes son la falta de desayuno, la frecuencia de comidas durante el día, el tamaño de las porciones y el consumo de alimentos "chatarra" y bebidas azucaradas ${ }^{8}$. En el presente trabajo se revelaron como factores protectores 
el hábito de desayunar y tener una frecuencia adecuada de comidas al día.

En estudios epidemiológicos se ha encontrado una tendencia creciente paralela entre la epidemia de obesidad y el consumo de bebidas azucaradas ${ }^{6,8}$. En Estados Unidos y México, países que sobresalen por la elevada prevalencia de obesidad en la adolescencia, el consumo de bebidas azucaradas contribuye un $10-12 \%$ de las calorías diarias.

Moreno ${ }^{8}$ plantea que, de todos los factores dietéticos, el único que en estudios longitudinales ha mostrado una clara asociación positiva con el desarrollo de la obesidad es el consumo de bebidas azucaradas. Los estudios prospectivos muestran una asociación positiva consistente entre el consumo de bebidas azucaradas y la ganancia de peso y la obesidad en niños, adolescentes y adultos ${ }^{6}$.

La falta de asociación entre la cantidad de azúcar añadida a los refrescos instantáneos, la leche, el yogur y los jugos de frutas y el exceso de grasa puede deberse a lo generalizado y frecuente de su consumo. Además, que sólo un 61-77\% diera respuesta de la cantidad de azúcar añadida podría constituir un sesgo en la falta de asociación encontrada.

El esquema de estilos de vida y hábitos alimentarios inadecuados que se describe frecuentemente en los adolescentes de otros países ${ }^{7,8,21}$ se repite en el presente estudio.

Algunos de los resultados encontrados en los adolescentes del estudio se asemejan a los hallados en preescolares ${ }^{30}$ y en adultos cubanos de la I Encuesta Nacional de Consumo y Preferencias Alimentarias que se realizó como parte de la II Encuesta Nacional de Factores de Riesgo de Enfermedades Crónicas del año 2001, en la que las mujeres resultaron más sedentarias, consumían más azúcar y tenían mayor exceso de peso que los varones (el 49 frente al 38\% (35. $^{35}$.

La escuela es el lugar idóneo para realizar intervenciones de educación nutricional en que estén involucrados no sólo los adolescentes, sino también los padres y los docentes. Para que estas resulten efectivas en la prevención y el tratamiento de la obesidad, deben integrar las acciones de promoción de la actividad física y la disminución de las actividades sedentarias. Conjuntamente deben realizarse acciones para promocionar una alimentación saludable, haciendo énfasis en la disminución de la cantidad de azúcar añadida y el consumo de refrescos.

\section{Conclusiones}

El exceso de peso, y especialmente el exceso de grasa, puede ser considerado un problema de salud en los adolescentes estudiados. Tanto las mujeres como los varones consumían una cantidad demasiado alta de azúcar, dedicaban un tiempo excesivo a ver TV, particularmente el fin de semana, y realizaban insuficiente actividad física, especialmente las mujeres.

\section{Agradecimientos}

A las técnicas Iraida Wong, Dayany Matos, Margarita Pavón por realizar las mediciones antropométricas. A la Lic. Yeneisy Lanyau y las técnicas Denia Reyes, Caridad Arocha y Ana Ferret por la aplicación de las encuestas. A las Lics. Luz
Divina Gallardo y Ruth García del Ministerio de Educación por el apoyo metodológico. A la oficina del UNICEF en Cuba por el apoyo financiero al proyecto.

\section{Conflictos de intereses}

No existen conflictos de intereses.

\section{Bibliografía}

1. World Health Organization. Diet, Nutrition and the Prevention of Chronic Diseases. WHO/FAO Expert Consultation: 916. Geneva: WHO; 2002.

2. Esquivel M, González C. Excess weight and adiposity in children and adolescents in Havana, Cuba: Prevalence and trends, 1972 to 2005. MEDICC Rev. 2010;12:13-8.

3. Jiménez Acosta $S$, Rodríguez Suárez A, Selva Suárez L, Martín Oramas E, González Guzmán E, Pérez Jiménez D. Sobrepeso en pre-escolares cubanos. Un análisis de la vigilancia nutricional pedíatrica mediante sitios centinela. Rev Esp Nutr Comunitaria. 2004;10:70-3.

4. DeMattia L, Lemont L, Meurer L. Do interventions to limit sedentary behaviours change behaviour and reduce childhood obesity? A critical review of the literature. Obes Rev. 2007;8: 69-81.

5. Martínez-Gómez D, Ruíz JR, Ortega FB, Veiga OL, MolinerUrdiales D, Mauro B, et al; on behalf of the HELENA Study Group. Recommended levels of physical activity to avoid an excess of body fat in European adolescents. Am J Prev Med. 2010;39:203-11.

6. Malik VS, Schulze MB, Hu FB. Intake of sugar-sweetened beverages and weight gain: a systematic review. Am J Clin Nutr. 2006;84:274-88.

7. Foltz JL, Cook SR, Szilagyi PG, Auinger P, Stewart PA, Bucher S, et al. US Adolescent Nutrition, Exercise, and Screen Time Baseline Levels Prior to National Recommendations. Clin Pediatr (Phila). 2011;50:424-33.

8. Moreno LA, Rodríguez G. Dietary risk factors for development of childhood obesity. Curr Opin Clin Nutr Metab Care. 2010;10:336-41.

9. Weiner JS, Lourie JA. Human biology: a guide to field methods. International Biological Programme. Handbook No 9. Oxford: Blackwell Scientific Publication; 1969. p. 3-59.

10. Boileau RA, TG Lohman, Slaughter MH. Exercise and body composition of children and youth. Scand J Sport. 1985;1:1727.

11. Esquivel M. Valores cubanos del índice de masa corporal en niños y adolescentes de 0-19 años. Rev Cubana Pediatr. 1991;63:181-90.

12. Esquivel $M$, Rubí A. Valores percentiles del área de músculo y grasa del brazo en niños y adolescentes de 0-19 años. Rev Cubana Aliment Nutr. 1990;4:70-85.

13. Deurenberg P, Pieters JIL, Hautuast JGL. The assessment of the body fat percentage by skinfold thickness measurement in childhood and young adolescent. Br J Nutr. 1990;63:23-24.

14. IPAQ. The International Physical Activity Questionnaire. Disponible en: http://www.ipaq.ki.se/

15. Macías C, Pita GM, Basabe B, Herrera D, Lanyau Y. Hábitos, actitudes y preferencias alimentarias en adolescentes de enseñanza media de La Habana. Rev Esp Nutr Comunit. 2009; 15:13-22.

16. Ogden CL, Carroll MD, Curtin LR, Lamb MM, Flegal KM. Prevalence of high body mass index in US children and adolescents, 2007-2008. JAMA. 2010;303:242-9. 
17. Moreno LA, Mesana MI, Fleta J, Ruiz JR, González-Gross M, Sarría A, et al. The AVENA Study Group. Overweight, obesity and body fat composition in Spanish adolescents. Ann Nutr Metab. 2005;49:71-6.

18. Lien N, Henriksen HB, Nymoen LL, Wind M, Klepp KI. Availability of data assessing the prevalence and trends of overweight and obesity among European adolescents. Publ Health Nutr. 2010;13:1680-7.

19. Poletti $\mathrm{OH}$, Barrios L. Sobrepeso, obesidad, hábitos alimentarios, actividad física y uso del tiempo libre en escolares de Corrientes (Argentina). Rev Cubana Pediatr. 2007;79.

20. Giugliano R, Carneiro EC. Factors associated with obesity in school children. J Pediatr (Rio J). 2004;80:17-22.

21. Turconi G, Guarcello M, Maccarini L, Cignoli F, Setti S, Bazzano R, et al. Eating habits and behaviors, physical activity, nutritional and food safety knowledge and beliefs in an adolescent Italian population. J Amer Coll Nutr. 2008;27:31-43.

22. Kurt BM, Schaffrath RA. Die Verbreitung von Übergewicht un Adipositas bei Kindern und Jugendlichen in Deutschland. Ergebnisse des bundesweiten Kinder und Jugendgesundheitssurvey (KIGGS). Bundesgesundheitsbl Gesundheitsforsch Gesundheitsschutz. 2007;50:736-43.

23. Olivares S, Kain J, Lera L, Pizarro F, Vio F, Morón C. Nutritional status, food consumption and physical activity among Chilean school children: a descriptive study. Europ J Clin Nutr. 2004; 58:1278-85.

24. Caballero C, Hernández B, Moreno H, Hernández-Girón C, Campero L, Cruz A, et al. Obesidad, actividad e inactividad física en adolescentes de Morelos, México: un estudio longitudinal. ALAN. 2007;57:231-7.

25. Lazarou C, Soteriades ES. Children's physical activity, TV watching and obesity in Cyprus: The CYKIDS study. Eur J Public Health. 2009;20:70-7.
26. Shroender DK, Martorell R. Fatness and body mass index from birth to young adulthood in a rural Guatemalan population. Am J Clin Nutr. 1999; Suppl:S137-44.

27. Ortiz L. Evaluación nutricional de adolescentes. Rev Med ISSM. 2002;40:153-62.

28. Lagarde F, Claire MA, LeBlanc MD. Policy options to support physical activity in schools. Can J Public Health. 2010;101 Suppl 2:S9-13.

29. Sarmiento O, Torres A, Jacoby E, Pratt M, Schmid TL, Stierling G. The Ciclovía-Recreativa: A mass-recreational program with public health potential. J Phys Act Health. 2010;7 Suppl 2: S163-80.

30. Hernández-Triana M. Fitness vs. Obesity in Cuban children: battling the biases of gender and geography. MEDICC Rev. 2010;12:48.

31. Russ SA, Larson K, Franke TM, Halfon N. Associations between media use and health in US children. Acad Pediatr. 2009;9: 300-6.

32. Taveras EM, Field AE, Berkey CS, Rifas-Shiman SL, Frazier L, Colditz GA, et al. Longitudinal relationship between television viewing and leisure-time physical activity during adolescence. Pediatrics. 2007;119:314-9.

33. Sisson SB, Broyles ST, Baker BL, Katzmarzyk PT. Screen time, physical activity, and overweight in U.S. youth: National Survey of Children's Health 2003. J Adolesc Health. 2010;47: 309-11.

34. Veerman JL, Van Beeck EF, Barendregt JJ, Mackenbach JP. By how much would limiting TV food advertising reduce childhood obesity? Eur J Public Health. 2009;19:365-9.

35. Díaz ME, Jiménez SM, García RG, Bonet M, Wong I. Overweight, obesity, central adiposity and associated chronic diseases in Cuban adults. MEDICC Rev. 2009;11:23-8. 